\title{
What if a child has never been immunized?
}

\author{
B Tan MD FRCPC ${ }^{1}$, L Nolan MD MHSc FRCPC ${ }^{2}$
}

C hildren in Canada are routinely immunized against nine infectious diseases: diphtheria, tetanus, pertussis, poliomyelitis, Haemophilus influenzae type b (Hib) infection, measles, mumps, rubella and hepatitis B. Vaccines for these diseases are administered following immunization schedules that vary slightly between provinces and territories (1). A varicella vaccine was licensed in Canada in December 1998 (2), but is not yet routinely used in most provincial or territorial childhood programs (Prince Edward Island is initiating a childhood varicella immunization program in 2000). Despite high overall vaccine coverage (estimated at $82 \%$ to $95 \%$ for individual vaccine antigens when assessed at 24 months of age [3]), there remain individuals or pockets of children in Canada who are susceptible to infection because they have never been or are incompletely immunized against one or more of these nine infectious diseases.

New immigrants from countries who have not had one or more of the above-mentioned childhood vaccines make up part of this never-immunized group, especially if 'catch-up' immunizations are not provided after their arrival in Canada (4). Some children are not immunized because they have valid medical contraindications (5), they missed immunization appointments or their parents declined to have the children immunized. Some parents have religious reasons for declining the immunizations, while others hold mistaken beliefs that the infectious diseases either do not cause serious harm or no longer occur in Canada (6). Infectious diseases that are rare in Canada, such as diphtheria and poliomyelitis, may be reintroduced into the country from abroad. Cases of measles, mumps, rubella, pertussis, tetanus, Hib and hepatitis B are still reported in Canada. Examples include the recent outbreaks of rubella in Manitoba (7) and mumps in British Columbia (8).

Because the immunization coverage in Canadian children is generally high, physicians may wrongly assume that children are fully immunized by the time that they enter school. Children's immunization records should be checked to ensure that the children's immunization are up-to-date whenever they are exposed to vaccine-preventable infections. Table 1 (middle column) outlines the preventive steps that physicians and public health authorities recommend when faced with a susceptible child who has been exposed to one of these infections. This often involves one or a combination of the following steps: using immune globulin preparations to provide immediate protection; starting the primary immunization series to provide either immediate (eg, postexposure immunization for measles, varicella and hepatitis A) or future protection; prescribing antibiotic prophylaxis; and excluding the child from school or daycare to prevent further exposure or spread of the infection. For fully immunized children, these steps may be eliminated (see Table 1, right-hand column for comparison).

Parents should be encouraged to inform their physicians when their unimmunized child is exposed to a vaccinepreventable illness so that the appropriate steps can be taken during a bona fide exposure. Unbeknownst to the parents, a susceptible child may be exposed to infectious persons who are asymptomatic or the child may sustain a wound that becomes contaminated with Clostridium tetani and tetanus may develop without an opportunity to provide preventive measures. Parents of unimmunized children are often unaware that a blood product (immune globulin) may be administered in the absence of prior immunity to certain infections. A 'catchup' primary immunization series that is initiated only after exposure has occurred will not usually induce immunity in time to prevent or reduce the severity of the infection (with the exception of measles, varicella and hepatitis A, where postexposure immunization is effective in preventing illness). Therefore, avoiding the primary series during infancy simply delays the immunization until after an infectious exposure has occurred.

Adults who were not immunized during childhood may travel abroad and be exposed to these vaccine-preventable infectious diseases in foreign countries. In developing countries, health care may not be readily available, thereby increasing the risk of morbidity or mortality from these infectious diseases. Furthermore, colleges offering medical, veterinary, dental, nursing and laboratory technology degrees require students to

\footnotetext{
All material presented in Paediatric Infectious Disease Notes has been reviewed by the Canadian Paediatric Society Board of Directors

${ }^{1}$ University of Saskatchewan, Royal Unversity Hospital, Saskatoon, Saskatchewan;

${ }^{2}$ Associate Medical Officer of Health, Waterloo Region, Waterloo, Ontario

Correspondence: Infectious Diseases and Immunization Committee, Canadian Paediatric Society, 2204 Walkley Road, Suite 100, Ottawa, Ontario K1G 4G8. Telephone 613-526-9397, fax 613-526-3332, web site www.cps.ca
} 
be immunized before beginning their courses. Unimmunized persons may be denied access into these occupational fields.

Parents should be informed that it is never too late to start or complete the immunization series because catch-up immunizations may be arranged following the schedules recommended by Canada's National Advisory Committee on Immunization (9). Parents who remain unconvinced that immunizations provide protection against these infectious diseases should be encouraged to explain to their older children that they were not immunized during childhood, and therefore are still susceptible to (if not already infected with) these infectious agents. In this case, when adults, these children may wish to make up their own minds about wanting protection against these infections, especially before international travel, starting college and contemplating a pregnancy (with potential risk of having a child with congenital rubella syndrome).

TABLE 1: Preventive steps for both unimmunized and fully immunized children after a close contact with a confirmed vaccinepreventable infectious disease

\section{Child ex-}

posed to a Unimmunized child (ie, the child is considered susceptible to case of the specified infectious disease) ${ }^{*,+}$

Diphtheria

- Perform throat swab culture for diphtheria to determine if the child has been infected.

- Prescribe erythromycin or penicillin to prevent (or treat) infection.

- Recommend the completion of the primary diphtheria immunization series for future protection.

- Give diphtheria antitoxin (equine) treatment only if the child is symptomatic with laryngitis, nasopharyngitis or tonsillitis (do not wait for the throat swab culture report as it may be too late to prevent myocarditis or neuritis). Children who have pharyngeal carriage of Corynebacterium diphtheriae but who are asymptomatic should receive antibiotic treatment but not antitoxin.

- Exclude the child from school or daycare until all known cases in the class are appropriately treated and no longer infectious; also exclude the child if he or she develops pharyngitis (until infection is ruled out). $\$$

Pertussis

- If the child develops a cough, collect nasopharyngeal aspirate for pertussis culture to determine if the child has been infected.

- Prescribe erythromycin prophylaxis for 10 days (this measure can prevent culture-positive pertussis in household contacts but not necessarily the paroxysmal cough). Some experts in Canada recommend erythromycin prophylaxis for seven days, based on more recent studies $(14,15)$. Currently, there are no adequate studies and hence no guidelines for the use of clarithromycin or azithromycin for prophylaxis against pertussis infection.

- Recommend the completion of the primary pertussis immunization series for future protection (the acellular pertussis vaccine is now licensed for use in persons over seven years of age in Canada; guidelines of the use of this vaccine are being developed by the National Advisory Committee on Immunizations).

- Exclude the child from school or daycare if the child develops a cough (until pertussis is ruled out or the child has completed at least five days of the erythromycin treatment). It is debatable whether the child should be excluded from school or daycare until all infected contacts are appropriately treated. $\$$

Tetanus - For wounds in which Clostridium tetani may have been introduced, administer tetanus immune globulin (TIG) to provide immediate protection.

- Recommend the completion of the primary tetanus toxoid series for ongoing protection. If the primary series is not started or completed, TIG may be necessary for every subsequent significant injury.

- Exclusion from school or daycare is not necessary.
Fully immunized child (ie, the child is considered immune or partially immune to the specified infectious disease $)^{t, \pm}$

- Give a dose of diphtheria toxoid, only if more than 10 years have passed since the last dose, to boost the immunity against diphtheria.

- Perform throat swab culture for diphtheria to determine if the child has been infected.

- Prescribe erythromycin or penicillin to prevent (or treat) infection, regardless of immunization status or age.

- Exclude the child from school or daycare (to protect the others) only if he or she develops pharyngitis until infection is ruled out.\$

- Give a dose of acellular pertussis vaccine as a booster if it has been more than three years after the last dose (guidelines for the use of acellular pertussis vaccine in persons over seven years of age are being developed by the National Advisory Committee on Immunization).

- Prescribe erythromycin prophylaxis for 10 days, regardless of the fact that the primary pertussis vaccine series was completed because the vaccine only provides partial protection and vaccinated individuals may harbour Bordetella pertussis. Some experts in Canada recommend erythromycin prophylaxis for seven days, based on more recent studies $(14,15)$. Currently, there are no adequate studies and hence no guidelines for the use of clarithromycin or azithromycin for prophylaxis against pertussis infection.

- Exclude the child from school or daycare only if the child develops a cough (until pertussis is ruled out or the child has completed at least five days of the erythromycin treatment).

- Give a booster of tetanus toxoid if it has been more than 10 years since the last dose (except when dealing with heavily contaminated wounds, where a booster is recommended if it has been more than five years since the last dose).

- TIG is not necessary for any category of wound (unless the child has an underlying immunodeficiency, where TIG use is then recommended).

- Exclusion from school or daycare is not necessary. 
TABLE 1: Preventive steps for both unimmunized and fully immunized children after a close contact with a confirmed vaccinepreventable infectious disease (continued)

Child exposed to Unimmunized child (ie, the child is considered susceptible to
a case of

Poliomyelitis

Haemophilus influenzae type b (Hib)

Measles (16)

- In Canada, the last case of paralytic poliomyelitis caused by to prevent gastrointestinal infection. the completion of the primary series with OPV instead is vaccine recipients and less so in their contacts. administered. $\$$

- If the child is younger than 48 months of age, antibiotic prophylaxis with rifampin for four days is recommended for

- Recommend the completion of the primary series of Hib shots for children under 15 months of age (or only one dose of Hib vaccine if the child is between 15 and 59 months of age). prophylaxis is administered. wild virus was reported in 1988. However, if an unimmunized child is in close contact with a case suspected of being caused by wild virus, a dose of oral polio vaccine (OPV) is preferred

- Recommend the completion of the previously unimmunized child's primary series with inactivated polio vaccine for future protection (unless an outbreak of the wild virus occurs where recommended). Note that OPV itself has a very small risk of inducing vaccine-associated paralytic polio, mainly in primary

- Exclude the child from school or daycare until OPV has been the susceptible child and all household members (because the susceptible child may acquire Hib from these family contacts).

- Exclusion from school or daycare is not necessary if antibiotic

- Recommend a single dose of measles vaccine within three days of the exposure (if not pregnant) - this is the preferred step. A second dose of measles vaccine may be administered one to two months after the first dose.

- Administer immune serum globulin (ISG) if it is within six days of exposure - this should not be given if vaccination (the preferred step) is performed.

- If measles vaccine was not administered and ISG was given, recommend a 'catch-up' measles vaccination five months after the ISG dose to provide future protection, with the second dose one to two months later.

- Exclude the child from school or daycare (eg, for at least two weeks and up to 21 days after the last classmate breaks out with measles if immunization with either measles vaccine or ISG is not given) to protect the child and the other children. $\S$

Mumps
Fully immunized child (ie, the child is considered immune or partially immune to the specified infectious disease) $)^{+, \neq}$

- A polio vaccine booster is not deemed necessary for a fully immunized child who is exposed to wild polio virus.

- Exclusion from school or daycare is not necessary.

- Antibiotic prophylaxis is not necessary for the child and household members, unless there is another child in the family who is younger than 12 months of age (regardless of immunization status) or between 12 and 48 months of age who is inadequately immunized.

- Exclusion from school or daycare is not necessary.

- Measles vaccine booster is not necessary if the child has received two doses of measles vaccine in the past.

- Consider administering a single booster dose of measles vaccine (if not pregnant) if the child has received only one prior dose.

- ISG is not necessary (unless the child has an immunodeficiency disease where the previous immunization with measles vaccine was likely ineffective).

- Exclusion from school or daycare is not necessary (unless the child is known to have an underlying immunodeficiency disorder).

- A mumps vaccine booster is not necessary if the child has received at least one dose of mumps vaccine in the past.

- Exclusion from school or daycare is not necessary. 
TABLE 1: Preventive steps for both unimmunized and fully immunized children after a close contact with a confirmed vaccinepreventable infectious disease (continued)

$\begin{aligned} & \text { Child exposed to a } \\ & \text { case of }\end{aligned}$
the specified infectious disease) $)^{*,+}$

Rubella - Immunization with rubella vaccine after exposure is not thought to prevent illness from this exposure, but it will protect against subsequent exposures. Therefore, recommend immunization (if not pregnant) with a single dose of rubella vaccine.

- ISG may prevent or reduce the severity of rubella in females of child-bearing age after a known exposure to an infectious case. In pregnant females, ISG may not prevent the fetus from being infected with rubella, with the subsequent risk of congenital defects if the infection occurs during the first trimester.

- Exclude females of child-bearing age from school (eg, for up to 21 days after the onset of illness in the last classmate with rubella) to protect the female and her classmates. $\$$

The exclusion of male children and girls below child-bearing age from school or daycare is debatable.

Hepatitis B
- The risk of infection is highest if the exposure involves a person sharing needles contaminated with hepatitis B virus from infected injection drug users or having sexual intercourse with a person with an acute case of hepatitis B or a hepatitis B carrier; or a baby being born to a mother who is a hepatitis B carrier. In known exposures, protection is provided by administering hepatitis B immune globulin (HBIG) and initiating the primary immunization series of three doses of hepatitis B vaccine.

- The risk of infection is lower for children who live in the same household with another person who is a hepatitis B carrier. $\mathrm{HBIG}$ is not necessary, but the completion of the primary series of three doses of hepatitis $B$ vaccine is recommended.
Fully immunized child (ie, the child is considered immune or partially immune to the specified infectious disease $)^{t, \neq}$

- A rubella vaccine booster is not necessary if the child has received at least one dose of rubella vaccine in the past.

- ISG is not necessary (unless the child has an immunodeficiency disease where previous immunization with rubella vaccine was likely ineffective).

- Exclusion from school or daycare is not necessary.
- After a known exposure to the blood or bodily fluids of an infected person, the child's serum hepatitis B surface antibody titre may be determined; if the titre indicates immunity to hepatitis B, HBIG is not necessary.

- Exclusion from school or daycare is not necessary.

*This column refers to a child who has not received any doses of the specified vaccine and is therefore considered susceptible. ${ }^{\dagger}$ Wherever possible, the primary immunization series or booster dose recommended should be given as a combination vaccine to give maximum benefit to the child (eg, if measles vaccine is recommended, provide measles-mumps-rubella vaccine; if tetanus or diphtheria toxoid is recommended, provide diphtheria, tetanus and acellular pertussis vaccine or tetanus and diphtheria toxoid vaccine, whichever is age appropriate). ${ }^{*}$ This column refers to a child who has received the full number of doses constituting the primary series of the vaccine (in most cases, three doses). The child is thus considered immune to the disease, except in situations where completion of the series only confers partial immunity (eg, pertussis). ${ }^{\$}$ School or daycare exclusion practices may vary considerably between public health jurisdictions; please consult your public health office for the local policies

\section{REFERENCES}

1. Routine childhood immunization programs in Canada. Paediatr Child Health 1998;3:20.

2. National Committee on Immunization (NACI). Statement on recommended use of varicella virus vaccine. Can Commun Dis Rep 1999;25(ACS-1).

3. Canadian National Report on Immunization, 1997. Paediatr Child Health 1998;3(Suppl B):23B.

4. Immunizations for new immigrant and refugee children. In: Tan B, Ford-Jones EL, MacDonald NE, et al (eds). Children and Youth New to Canada: A Health Care Guide. Ottawa: Canadian Paediatric Society, 1999:33-8.

5. General cautions and contraindications to vaccinations. In: National Advisory Committee on Immunization. Canadian Immunization Guide, Fifth Edition, 1998. Ottawa: Health Canada, 1998:4-8.

6. Gold R. Your Child's Best Shot: A Parent's Guide to Vaccination. Ottawa: Canadian Paediatric Society, 1997:7-8;117-35.

7. Macdonald A, Petaski K. Outbreak of rubella originating among high-school students - Selkirk, Manitoba. Can Commun Dis Rep 1997;23-13:97-101.

8. Bell A, Fyfe M, Bigham M, Daly P, Buxton J, Craig C. Outbreak of mumps among young adults - Vancouver, British Columbia. Can Commun Dis Rep 1997;23:169-72.

9. Recommended immunizations for infants, children and adults (including 'catch-up' schedules). In: National Advisory Committee on Immunization. Canadian Immunization Guide, Fifth Edition, 1998. Ottawa: Health Canada, 1998:45-7.

10. National Advisory Committee on Immunization. Canadian Immunization Guide, Fifth Edition, 1998. Ottawa: Health Canada, 1998.

11. Peters G, eds. 1997 Red Book Report of the Committee on Infectious Diseases (Red Book Report of the Committee on Infectious Diseases, 1997), 24th edn. Elk Grove Village: American Academy of Pediatrics, 1997.

12. Advisory Committee on Epidemiology and the Division of Immunization, Laboratory Centre for Disease Control, Health Canada. Guidelines for the control of diphtheria in Canada. Can Commun Dis Rep 1998;24S3(Suppl).

13. National Advisory Committee on Immunization, Advisory Committee on Epidemiology and the Canadian Paediatric Society. Statement on management of persons exposed to pertussis and pertussis outbreak control. CMAJ 1995;152:712-6.

14. Halperin SA, Bortolussi R, Langley JM, et al. A randomized, placebo-controlled trial of erythromycin estolate chemoprophylaxis for household contacts of children with culture-positive Bordetella pertussis infection. Pediatrics 1999;104:e42.

15. Halperin SA, Bortolussi R, Langley JM, Miller B, Eastwood BJ. 
Seven days of erythromycin estolate is as effective as fourteen days for the treatment of Bordetella pertussis infections. Pediatrics 1997;100:65-71.
16. Guidelines for control of measles outbreaks in Canada (revised 1995). Advisory Committee on Epidemiology. Can Commun Dis Rep 1995;21(21):189-95.

\section{INFECTIOUS DISEASES AND IMMUNIZATION COMMITTEE}

Members: Drs Upton Allen, The Hospital for Sick Children, Toronto, Ontario; François Boucher, Département de pédiatrie, Centre Hospitalier Universitaire de Québec, Pavillon CHUL, Sainte-Foy, Québec; H Dele Davies, Division of Infectious Diseases, Alberta Children's Hospital, Calgary, Alberta; Gilles Delage, Laboratoire de santé publique du Québec, Sainte-Anne-de-Bellevue, Québec (chair); Joanne Embree, The University of Manitoba, Winnipeg, Manitoba; Mireille Lemay, Department of Infectious Diseases, Sainte-Justine Hospital, Montréal, Québec; Charles Morin, Complexe hospitalier Sagamie, Chicoutimi, Québec (director responsible); David Speert, Division of Infectious and Immunological Diseases, University of British Columbia, Vancouver, British Columbia; Ben Tan, Division of Infectious Diseases, Royal University Hospital, University of Saskatchewan, Saskatoon, Saskatchewan

Consultants: Drs Noni MacDonald, Faculty of Medicine, Dalhousie University, Halifax, Nova Scotia; Victor Marchessault, Cumberland, Ontario Liaisons: Drs Susan King, Division of Infectious Diseases, The Hospital for Sick Children, Toronto, Ontario (Canadian Paediatric AIDS Research Group); Scott Halperin, Department of Pediatrics, IWK-Grace Health Centre, Halifax, Nova Scotia (IMPACT); Monique Landry, Direction de la santé publique de Laval, Laval, Québec (Public Health); Larry Pickering, Centre for Paediatric Research, Norfolk, Virginia (American Academy of Pediatrics); John Waters, Alberta Health, Edmonton, Alberta (Epidemiology)

This article also appears in Paediatr Child Health 2000;5(3):151-155.

\section{BOOK REVIEWS}

Clinical Infectious Diseases. A Practical Approach. Editor-in-chief: Richard K Root, Editors: Francis Waldvogel, Lawrence Corey, Walter Stamm (1999). Oxford University Press, Canadian Branch, 70 Wynford Drive, Don Mills, Ontario M3C 1 J9. ISBN 0-19-508103-x; 1013 pages; \$207.50.

Clinical Infectious Diseases. A practical approach is an excellent clinical reference book that is highly recommended for the noninfectious disease medical or paediatric specialists or family practitioners who care for patients with primary infectious disease problems or complications. It is also a very useful teaching aid for the academic infectious disease specialist to use as a background for lectures and tutorials, and in discussions with residents and students.

As its title suggests, it does indeed provide a very practical approach to solving infectious disease problems. The information presented is practical, and the set-up of the book and the individual chapters make the book very easy to use as a reference text. Initially, there are sections that provide useful background information concerning pathophysiology, diagnostic methods, antimicrobial drugs and vaccines. The chapters in these sections are all very well written, and the subjects are presented in a manner such that they are easy to understand. The chapters describing the approaches to infectious diseases are then grouped according to disease syndromes or infections in special patient or risk groups. There is one final section devoted to HIV/AIDS, which is appropriate considering the impact of this disease on patient care. Again, the chapters are all well written, and provide clear, concise and complete information, a task accomplished by having annotated biographies rather than formal referencing and by the extensive use of tables. The tables in this book are fantastic. It is by the use of tables and lists that the authors are able to provide so much relevant information so concisely. In many instances, the authors have provided data in their tables that it would take practitioners hours to find and collate themselves; hence, part of the usefulness of this book as a reference text.

Overall, the text is up-to-date, inclusive of the chapters on HIV related issues. In part, this is due to the fact that the authors are providing general approaches to problems. These are not usually subject to the rapid changes as are more specific recommendations. Also, the authors of the chapters are experts in their fields and have usually anticipated the changes that are likely to occur in their field of interest. The specific recommendations provided reflect this. The one drawback with the book is that the number of references is severely limited, and as a result the references are highly selective. Although this could potentially lead to a biased approach to some problems, I did not detect this when reviewing the book.

In summary, this book is a useful and needed reference textbook and is recommended for the noninfectious disease specialist medical or paediatric practitioner.

\footnotetext{
Joanne Embree MD FRCPC University of Manitoba Winnipeg, Manitoba
} 


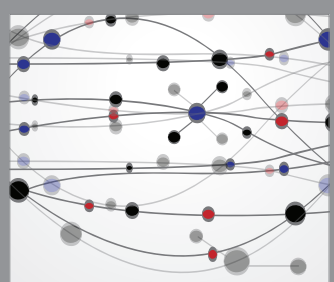

The Scientific World Journal
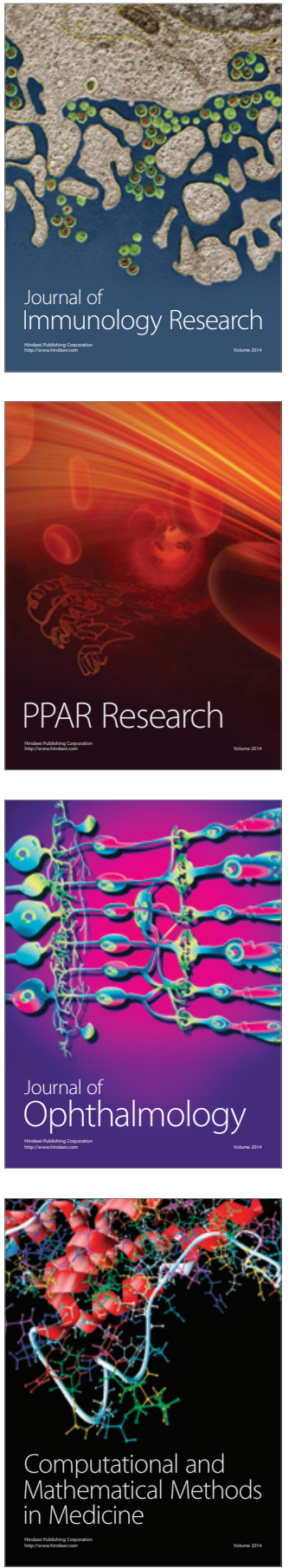

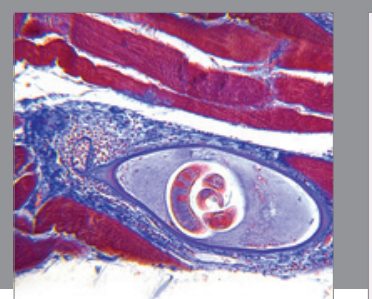

Gastroenterology Research and Practice

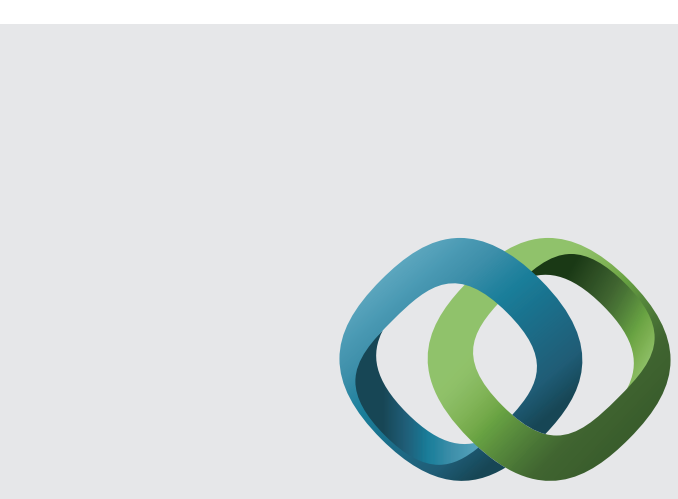

\section{Hindawi}

Submit your manuscripts at

http://www.hindawi.com
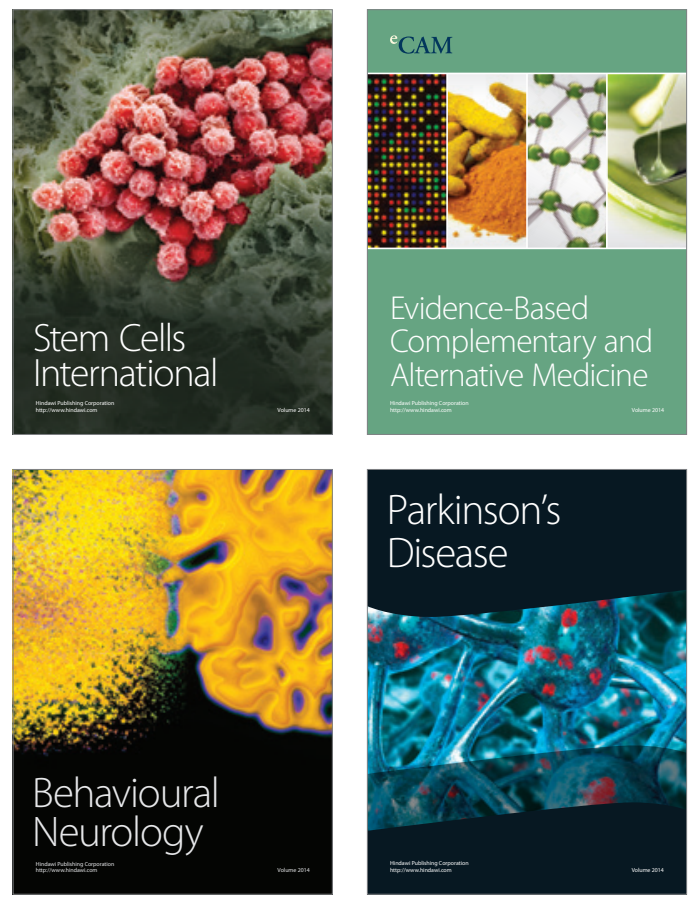
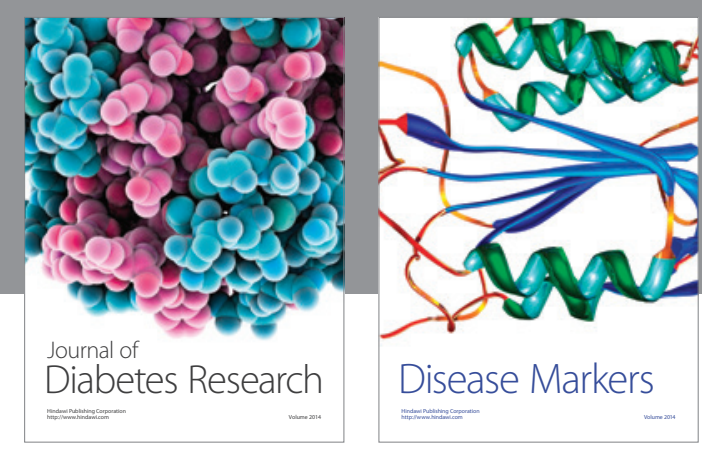

Disease Markers
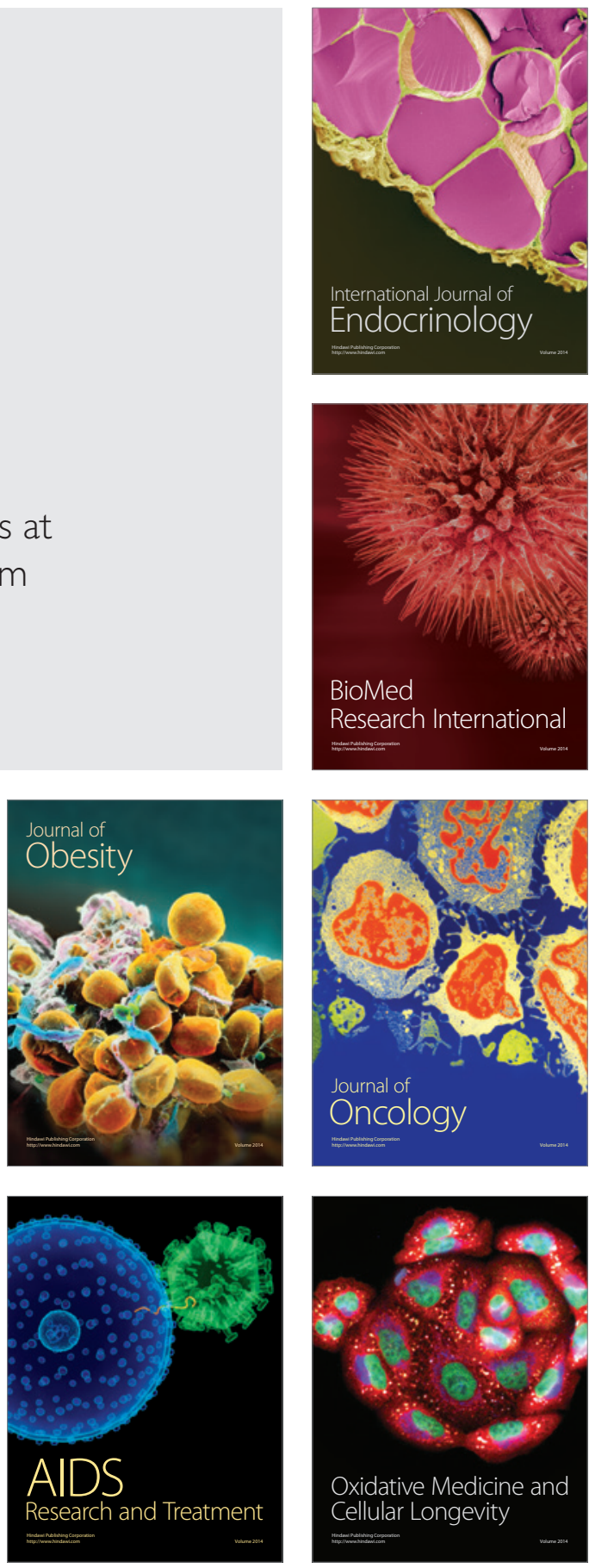